\title{
Collimation Optimization in High Intensity Rings *
}

\author{
N. Catalan-Lasheras ${ }^{\dagger}$ \\ Brookhaven National Laboratory, Upton, New York, 11973, USA.
}

\begin{abstract}
In high intensity proton rings, collimation is needed in order to maintain reasonable levels of residual activation and allow hands-on maintenance. Small acceptance to emittance ratio and restrained longitudinal space become important restrictions when dealing with low energy rings. The constraints and specifications when designing a collimation system for this type of machine will be reviewed. The SNS accumulator ring will serve as an example along which we will illustrate the optimization path. Experimental studies of collimation with $1.3 \mathrm{GeV}$ proton beams are currently under way in the U-70 machine in Protvino. The first results will be presented.
\end{abstract}

\section{INTRODUCTION}

In high intensity rings, the size of the beam is enhanced to avoid excessive space-charge forces. The beam thus occupies the highly nonlinear region of the magnetic field near the bore and most of the RF bucket. Large fringe fields are inherent to short and wide magnets and decrease further the beam stability [1]. Rapid acceleration produces non-adiabatic trapping on the longitudinal plane. All these effects pump protons from the core of the beam into the tails in all three dimensions. On the other hand, the relative aperture is dramatically small. While in a high energy collider the aperture of the accelerator is around $10-15 \sigma$ (where $\sigma$ is rms beam size), in a high intensity ring the aperture is typically less than $4 \sigma$.

A small fraction of the beam (0.01-0.1\%) in the tails lost in the machine corresponds to kilowatts of beam power. Residual and prompt radiation in the tunnel and working areas as well as air and soil activation are important concerns. Potential damage to the components in the machine also limits the high availability and reliability required. Collimation systems become essential to remove the tails of the beam in a controlled way $[2,3]$. By concentrating the beam losses in the collimators, low radiation levels and hands-on maintenance in the rest of the ring are guaranteed.

Besides the beam power, the main difference between high intensity machines and other existing machines where collimation systems are in place, arises from a lower beam energy. Section 2 explores the impact of low-medium energy range in the design and efficiency of the collimation systems. Section 3 focuses in the existing and proposed high intensity machines, their different constraints and the way beam losses are handled. The system chosen for the

\footnotetext{
* Work performed under the auspices of the U.S. Department of Energy
}

$\dagger$ catalan@bnl.gov
Spallation Neutron Source (SNS) is described in depth in Section 4. At last, Section 5 presents experimental studies on collimation that confirm and benchmark the assumptions taken when designing collimation systems.

\section{SPECIAL CONSTRAINTS}

High intensity machines have typically a beam kinetic energy of some GeV. While the engineering design of scrapers and collimators has to be able to handle high beam power and be designed accordingly, from the beam dynamics point of view, the low energy of the beam is the main issue and drives the design of the system.

\subsection{Aperture}

In general the beam coming from the source goes through partial or total acceleration after which it is accumulated in a ring to achieve the adequate peak intensity and time structure. In order to avoid the damaging effects of space-charge, the accumulated beam is painted in the three dimensional phase-space to an emittance much larger than the initial beam. One of the consequences of phase-space painting is that it requires also a large admittance from the accelerator. When multi-turn collimation is planned, the aperture has to be even larger to ensure the survival of outscattered particles and guarantee a good efficiency.

In a clear difference with high energy machines, an advantage of large beam emittance is that the alignment of the collimators and the control of the closed orbit is not so critical. A fraction of a millimeter is still a small fraction of the total beam size. The tolerance in the collimators is also less stringent and larger collimators can be manufactured. The impact parameter in the collimators is in general much bigger because of larger emittances.

\subsection{Coulomb Scattering}

When the proton beam passes through the collimation elements, protons suffer multiple Coulomb scattering (mCs) from the medium nucleus. Approximated by a Gaussian angular distribution, the rms scattering angle after traversing a target of thickness $x$ is given by the expression [4]:

$$
\theta_{0}=\frac{13.6 \mathrm{MeV}}{\beta c p} \sqrt{\frac{x}{\chi_{0}}\left(1+0.038 \ln \frac{x}{\chi_{0}}\right)}
$$

where $p$ and $\beta c$ are the momentum and velocity of the proton and $\chi_{0}$ is the radiation length of the material wich is a function of $Z$ and $A$ [4]. For the same collimator material, we see from Eq. 1 that lower energy produces larger 
scattering angles. The first drawback is the increase on the probability of being scattered outside the collimator when the impact parameter is small.

Fig. 1 shows the exit coordinate along the inner face of a collimator for two beams of energy $1 \mathrm{GeV}$ and $8 \mathrm{TeV}$ respectively. The impact parameter of the low energy beam has been multiplied by $10^{3}$ (similar to the ratio between $\beta \gamma$ at both energies). However, the fraction of out-scattered protons in the beginning of the collimator block is much larger in the low energy beam due to the large mCs angle.

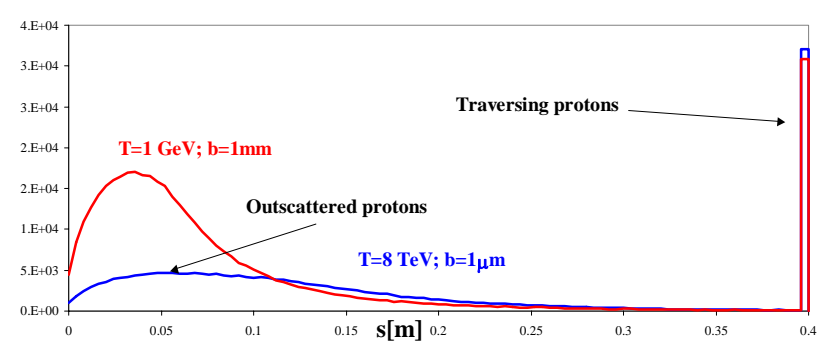

Figure 1: Distribution of the exit coordinate of $1 \mathrm{Gev}$ and $8 \mathrm{TeV}$ beams when traversing a $40 \mathrm{~cm}$ block of copper. Data from a Monte-Carlo simulation.

\subsection{Nuclear Scattering}

The removal of the protons from the beam is done trhough inelastic nuclear scattering with the nuclei of the collimator material. The inelastic cross section reaches a minimum around $\mathrm{E}=1 \mathrm{GeV}$ [4]. The capture efficiency is thus lower for low energy beams.

At the same time, the elastic nuclear cross section increases for lower energy adding significantly to the effect of multiple Coulomb scattering and increasing even more the probability of out-scattering (see Fig. 2).
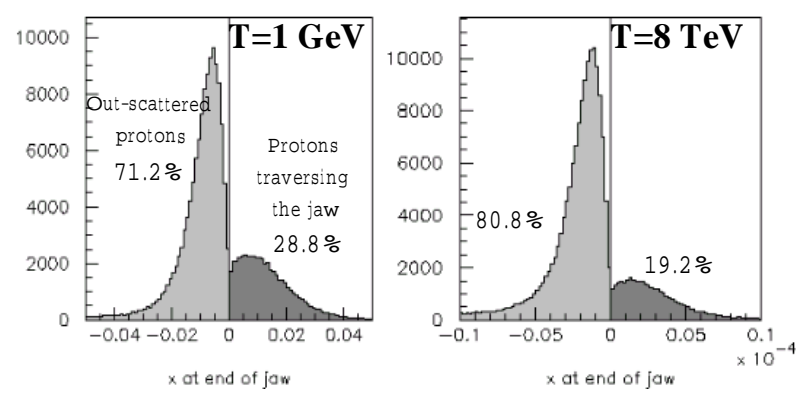

Figure 2: Final horizontal beam distribution of $1 \mathrm{GeV}$ and 8 $\mathrm{TeV}$ proton beam after $40 \mathrm{~cm}$ of copper. The impact parameter of both beams has been adjusted to have the same final efficiency $(82 \%)$. In both cases the first contribution to the inefficiency is out-scattering. At low energy, the block is more transparent to the passage of protons.

\subsection{Energy loss}

An important process taking place in the collimator is energy loss by ionization. The mean rate of energy loss is given by the Bethe-Bloch equation:

$$
-\frac{d E}{d x}=K z^{2} \frac{Z}{A} \frac{1}{\beta^{2}}\left[\frac{1}{2} \ln \frac{2 m_{e} c^{2} \beta^{2} \gamma^{2} T_{\max }}{I^{2}}-\beta^{2}\right]
$$

$\mathrm{K}$ is a constant, $m_{e}$ the electron mass and $I \approx(10 \pm 1 \mathrm{eV}) \cdot \mathrm{Z}$ the mean excitation energy. $T_{\max }$ is the maximum kinetic energy that can be imparted to a free electron in a single collision and is only a function of the proton energy. We have not included the density correction term, which is important only at very high energies. The energy loss of protons has a shallow minimum at $p=3 \mathrm{GeV} / \mathrm{c}$. It is nearly constant toward higher energues but rises fast bellow $1 \mathrm{GeV} / \mathrm{c}$. At $\mathrm{T} \approx 1 \mathrm{GeV}$ the relative energy loss by ionization is considerable. Protons out-scattered from the jaw and, even more, those traversing the full collimator may end outside the RF bucket acceptance or the momentum acceptance of the ring given by the maximum dispersion. Energy loss may help increasing inelastic scattering cross section as well as stopping completely the proton in the collimator. It does not help however against out-scattering in the beginning of the jaw.
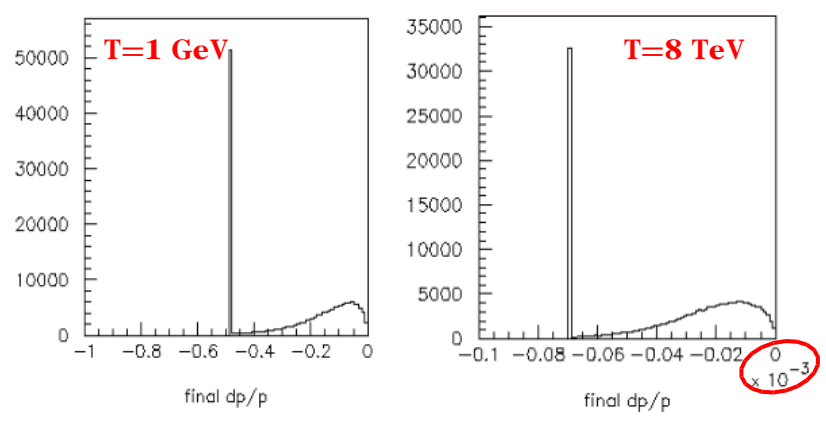

Figure 3: Final distribution of $\delta p / p_{0}$. Protons with kinetic energy of $1 \mathrm{GeV}$ loose up to half of their momentum by ionization.

If we adjust the thickness of the scraper to have a constant relative momentum loss of $d p / p_{0}=1 \%$, the rms angle given by Coulomb scattering increases with the atomic number of the target as shown in Fig. 4. High $\mathrm{Z}$ materials are preferred in order to increase the impact parameters in the secondary collimators with minimum energy loss.

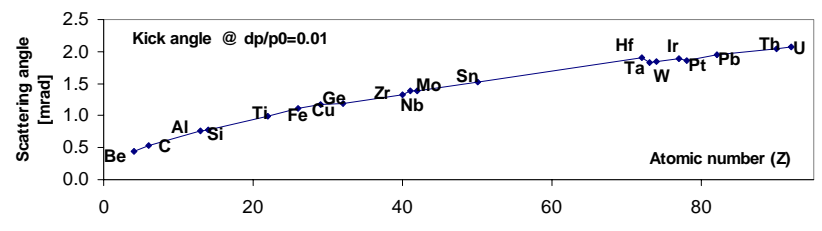

Figure 4: Scattering angle produced by mCs for different materials. The thickness of the scraper has been adjusted to loose $1 \%$ of the initial momentum according to Eq. 2.

Special care has to be taken when designing momentum collimation with scrapers. The energy loss in the target adds up to the negative momentum and prevents multi-turn collimation. Also, the betatron oscillation of negative momentum deviation protons may be damped [5]. 


\begin{tabular}{|l|c|c|c|c|c|c|c|}
\hline Machine & $\begin{array}{c}\mathrm{T} \\
{[\mathrm{GeV}]}\end{array}$ & $\begin{array}{c}\text { Power } \\
{[\mathrm{MW}]}\end{array}$ & $\begin{array}{c}\text { Loss } \\
{[\%]}\end{array}$ & $\beta$-collimation & z-collimation & $\begin{array}{c}\text { Aperture } \\
{[\pi \mathrm{mm} \cdot \mathrm{mrad}]}\end{array}$ & $\begin{array}{c}\text { Efficiency } \\
{[\%]}\end{array}$ \\
\hline RAL ISIS & 0.8 & 0.16 & 11.0 & 3 & $1+2$ & 410 & 80 \\
\hline LANL PSR & 0.8 & 0.8 & 0.3 & - & - & 140 & - \\
\hline \hline ORNL SNS & 1.0 & 2.0 & 0.1 & $1+2$ & BIG & 480 & $>97$ \\
\hline Japan JKJ & 3.0 & 1.0 & & \multicolumn{2}{|c|}{$2+8$} & & 93.9 \\
\hline ESS & 1.3 & 2.5 & 0.02 & $1+3$ & $1+2$ & 480 & $>95$ \\
\hline FNAL Proton Driver & 16.0 & 1.2 & 1.0 & \multicolumn{2}{|c|}{$2+3+5$} & - & 99 \\
\hline BNL Proton Driver & 24.0 & 1.0 & 3.0 & - & - & 55 & - \\
\hline
\end{tabular}

Table 1: Main collimation parameters of existing and planned high intensity rings. From left to right, kinetic energy, actual or projected beam loss, betatron and longitudinal collimation system, ring aperture and cleaning efficiency. [8,9]

\section{PAST EXPERIENCE}

Different machines take different approaches depending not only on requirements but also available space, energy or expertise. Two existing high intensity proton machines, namely ISIS in Rutherford Appleton Laboratory and Proton Storage Ring (PSR) at Los Alamos, have completely different approaches in dealing with beam losses and collimation. On one side, ISIS [6], a rapid cycling synchrotron with $0.16 \mathrm{MW}$ of beam power uses a multi-stage collimation system consisting of thin graphite/copper scrapers to absorb mainly trapping losses at low energy (up to $10 \%$ at $70 \mathrm{MeV}$ ). The measured efficiency reaches $80 \%$. After acceleration, losses become much less important but do not vanish. An upgrade has taken place in the collimator systems to accommodate losses at higher energy.

On the other side, the PSR is an accumulator ring storing $800 \mathrm{MeV}$ protons for a total beam power of $0.8 \mathrm{MW}$. In this case, no collimators are provided and the total losses are reduced by means of machine conditioning. A new $H^{-}$ injection and a tight control on instabilities have reduced the beam losses down to $0.3 \%$ of the total beam [7].

When designing new machines, where both the energy and the power increase considerably, none of these approaches would be sufficient by itself. Limiting and controlling beam losses will both become necessary to achieve reasonable beam loss levels and low radiation. In table 1, we indicate the main parameters of existing and planned high intensity proton rings.

\section{THE SNS CASE}

With a beam power of up to $2 \mathrm{MW}$ and a circumference of $248 \mathrm{~m}$, the beam loss limit of $1 \mathrm{~W} / \mathrm{m}$ translates into a fractional loss of around $10^{-4}$. With an expected halo of about $10^{-3}$ mainly due to space-charge, the collimation system of SNS must have an efficiency larger than $90 \%$ [10].

The optimization of the two-dimensional collimation system of the SNS is done by minimizing the escaping halo extent attending to the lattice functions and collimators geometry. For the present lattice of the SNS accumulator ring and using one primary and two secondary collimators the maximum extent of the residual halo was found to be $480 \pi \mathrm{mm} \cdot \mathrm{mrad}$. Realistic simulations including scattering in the collimator material show indeed that the residual halo above this amplitude satisfies the tight uncontrolled loss requirements. Our first requirement is to provide an overall aperture equal or bigger than $480 \pi$ $\mathrm{mm} \cdot \mathrm{mrad}$ to maintain the uncontrolled loss under reasonable limits. For momentum cleaning, we require a momentum acceptance of $d p / p_{0}= \pm 2 \%$ to allow the off-bucket beam to drift between bunches. The condition is easily satisfied along the straight sections. In the arc, the minimum betatron acceptance for this momentum deviation is $220 \pi \mathrm{mm} \cdot \mathrm{mrad}$, still larger than the beam emittance after painting to $160 \pi \mathrm{mm} \cdot \mathrm{mrad}$.

\subsection{Layout}

In the SNS accumulator ring, a straight section $30 \mathrm{~m}$ long is dedicated to transverse collimation. The lattice functions are common to all the machine and can not be modified inside the cleaning section. A set of four movable scrapers made out of $0.5 \mathrm{~cm}$ platinum plates acts as primary collimator increasing the divergence of the halo protons. Two heavy absorbers presenting an equivalent mass close to $60 \mathrm{~cm}$ of stainless steel are located downstream as fixed aperture secondary collimators $[8,11]$. The first secondary collimator is located in the long straight section between doublets. The middle point of the collimator has a phase advance from the primary of $\mu_{x}=43^{\circ}, \mu_{y}=26^{\circ}$. The last secondary collimator is located in the third straight section just before the matching quadrupole and the arc. Its phase advance with respect to the primary is $\mu_{x}=144^{\circ}, \mu_{y}=$ $161^{\circ}$. If needed, a third secondary collimator can be located at a phase advance of $\mu_{x}=54^{\circ}, \mu_{y}=42^{\circ}$.

Two painting schemes are planned for the SNS, which provide a circular or square beam in real space. The final size of the beam is likely to be modified during operation. The four platinum scrapers can move independently to adjust to any beam shape and size as illustrated in Fig. 5. The aperture of the primary collimator is given by the size of the beam and can be set between 120$300 \pi \mathrm{mm} \cdot \mathrm{mrad}$. The aperture of the secondary collimators is set such so ad to prevent it from becoming a primary collimator under nominal conditions Nominal apertures are $\epsilon_{x}=\epsilon_{y}=140 \pi \mathrm{mm} \cdot \mathrm{mrad}$ for a square cut and 
$\epsilon_{r}=160 \pi \mathrm{mm} \cdot \mathrm{mrad}$ for circular beam. Secondary absorbers have a fixed aperture $\epsilon_{2}=300 \pi \mathrm{mm} \cdot \mathrm{mrad}$.

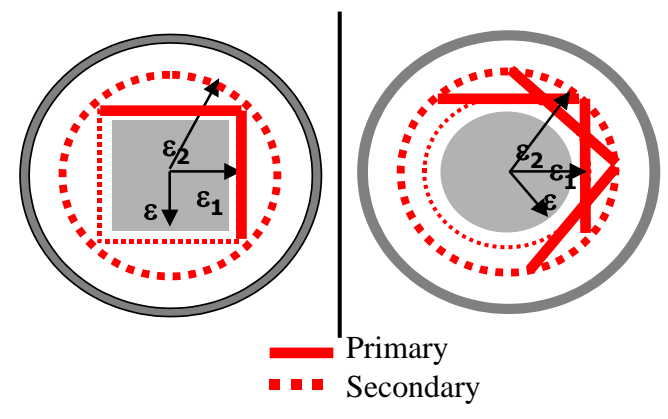
a) $\varepsilon_{1}>\varepsilon=120 ; \varepsilon_{2}>2 \varepsilon_{1}$
b) $\varepsilon_{1}>\varepsilon=160 ; \varepsilon_{2}>\varepsilon_{1}$

Figure 5: Schematic view of the primary and secondary collimators setup for correlated and anti-correlated painting.

The secondary collimators designed for the ring and transfer lines of the SNS consist of a layered structure designed to capture the beam protons and any resulting radioactive isotopes within the structure of the collimator.

\subsection{Efficiency}

Using the collimation system described in the previous section, we estimate the collimation efficiency using the K2 code, developed at CERN [12]. The code includes energy loss, multiple Coulomb scattering, nuclear elastic and inelastic scattering and has recently been adapted to low energy protons. Fig. 6 shows a survival plot for the actual baseline design of the collimation system of the SNS ring. We plot the fraction of the initial halo who escapes the collimation section without being captured above a given emittance $\eta(A)$. In a conservative approach we define the efficiency of the system as $\varepsilon=1-\eta(A)$ where $\mathrm{A}=$ $480 \pi \mathrm{mm} \cdot \mathrm{mrad}$ is the admittance of the ring. Both correlated and anti-correlated painting and cleaning schemes are presented. We obtain efficiencies larger than $90 \%$ for both nominal cases using two scrapers at $\epsilon_{1}=140 \pi \mathrm{mm} \cdot \mathrm{mrad}$ or four scrapers at $\epsilon_{1}=180 \pi \mathrm{mm} \cdot \mathrm{mrad}$. The final efficiency in both cases are $95 \%$ and $93 \%$, respectively. As a second figure of merit of the collimation system, we estimate the distribution of losses along the cleaning section.

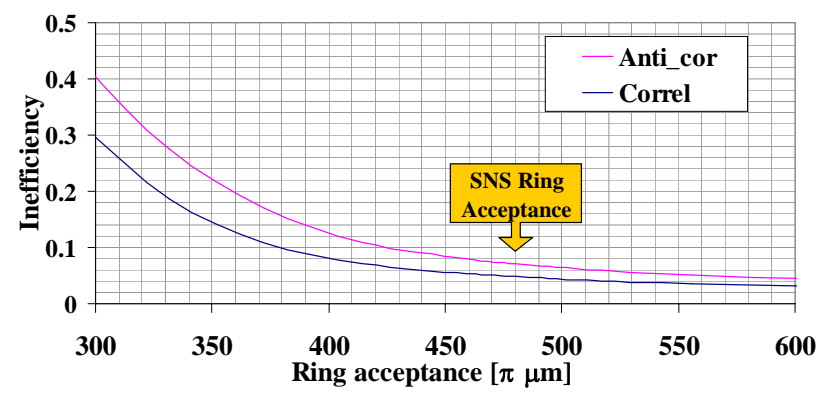

Figure 6: Residual halo profile after collimation for correlated and anti-correlated schemes.
To probe losses, we set completely absorbent collimators within every meter of free drift with an aperture equivalent to the vacuum pipe. We also place collimators at the entrance of each quadrupole. The results of the simulation are shown in Fig. 7. Based on these results, the structure around the scraper has been redesigned and shielding has been added before the QHB10 magnet to avoid damaging the quadrupole.

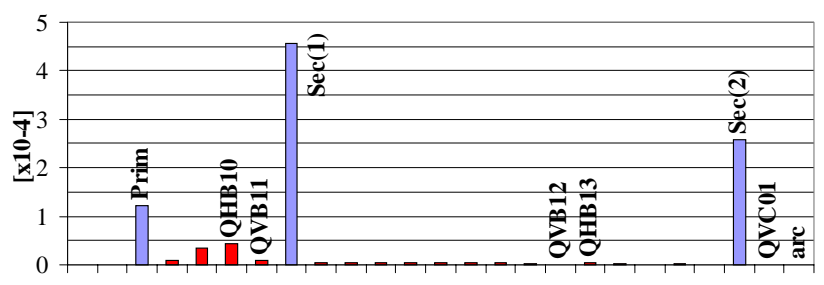

Figure 7: Beam loss distribution along the collimation straight section

\subsection{Momentum collimation}

As the space in the arcs is limited and the straight sections are dispersion free, there is not enough space in the ring to provide a dedicated momentum cleaning section using conventional collimators [5]. A solution is to install a fast rise kicker that fires between bunch passages. The kicker drives the protons to the collimation system in several turns (typically 10-20) where they are finnaly removed. The final absorption efficiency of these protons in the collimators is at least as high as for betatron losses because of larger impact parameters.

This principle has already been experimentally demonstrated in the National Synchrotron Light Source (NSLS) at Brookhaven and at HERA in DESY. The main difference is that the kicker polarity can be adjusted turn by turn following the betatron oscillation of the beam in gap. The sequence of kicks has to be resonant with the betatron tune to avoid damping of the amplitude (see [13] for complete references).

\section{EXPERIMENTAL STUDIES}

\subsection{CERN SPS studies at $120 \mathrm{GeV}$}

During 1996-97, a series of experiments was carried out at the SPS accelerator at CERN to benchmark the codes used to evaluate the efficiency of collimation system [14]. We arranged three pairs of movable aluminium blocks as primary, secondary and tertiary collimators and scintillation detectors next to them to measure the absorption rate on each one of them. By introducing white noise in a damper kicker, the size of a coasting beam was slowly increased and some $10^{10}$ protons per second were lost in the collimators producing enough signal for accurate measurements. The proton beam energy was $120 \mathrm{GeV}$.

With fixed aperture for the first $\left(A_{1}\right)$ and third $\left(A_{3}\right)$ of the collimators the second aperture $\left(A_{2}\right)$ was varied in the 
interval $\left[A_{1}, A_{3}\right]$. The counting rate in the scintillators for every collimator was recorded and compared with the simulation data for the same setting. The comparison between simulated and measured data on the SPS collimation experiment is shown in Fig. 8.

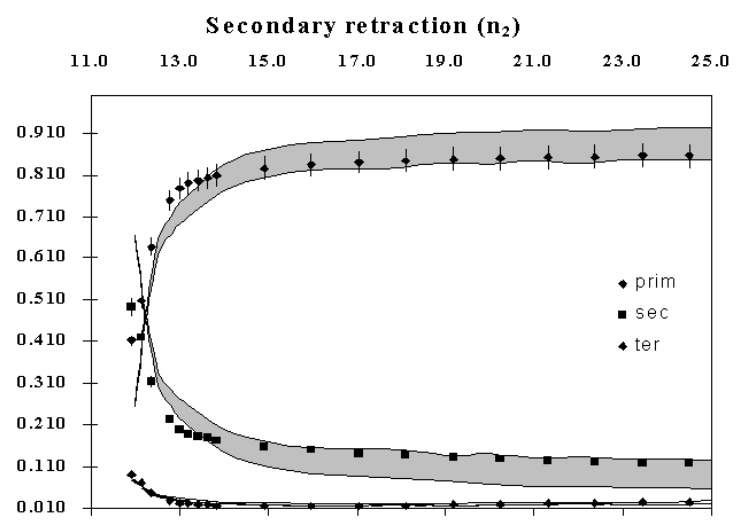

Figure 8: Proton absorption rate in all three collimators as a function of secondary jaws retraction. Simulation is represented by wide bands where protons lost in the pipe near the collimators are added to the signal with a weight between 0 and 1 .

\subsection{IHEP U70 studies at $1.3 \mathrm{GeV}$}

A series of experiments aiming to bench-mark the simulation codes at energy closer to $1 \mathrm{GeV}$ have been launched by the SNS project in collaboration with the Institute for High Energy Physics (IHEP) at Protvino, Russia [15].

The U-70 ring at Protvino is a twelve-superperiod proton synchrotron with an injection energy of $1.3 \mathrm{GeV}$ and a final energy of $70 \mathrm{GeV}$. Immediately after injection, half a second is available for measurements at low energy. A two meters collimator block made of stainless steel lays between magnetic units 85 and 86 . The collimator, of rectangular aperture, can be tilted in steps of $0.05-0.1 \mathrm{mrad}$ within a $\pm 3 \mathrm{mrad}$ range. Supports for movable thin targets or bending crystals are available at the beginning and at the end of the collimator straight section. We performed measurements with Carbon, Silicon and Tungsten targets as well as bent crystals.

We drive the beam into the block by exciting a dipole magnetic field in the combined magnets and producing a local bump at the collimator location. Eight beam loss monitors (BLM) are located along the next superperiod following the collimator. Two scintillators are located upstream from the collimator to measure the backscattered hadronic shower. A profile monitor in the front face of the collimator allows to measure the impact parameter on the front face with a precision of $1 \mathrm{~mm}$. The beam intensity is measured with current transformers to normalize different measurement data to the actual loss. A system of beam loss monitors around the ring allows to spot other loss locations and verify that we are not merely shifting the losses to another location.

The first confirmation of the principles exposed here is

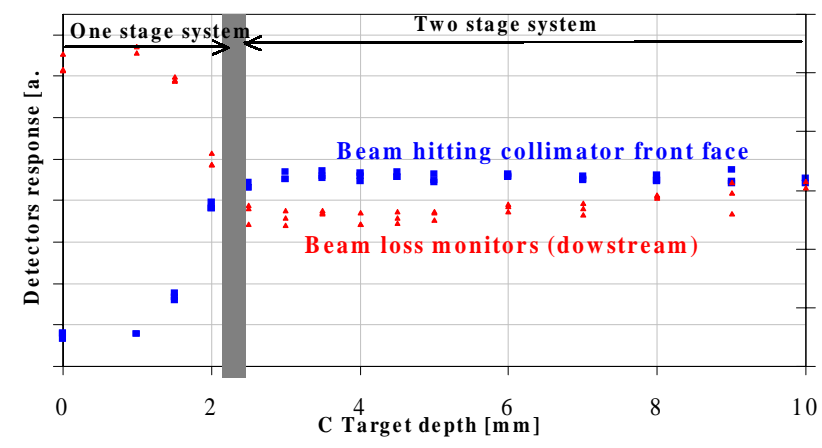

Figure 9: Detectors signal when the carbon target is introduced in the beam and becomes the primary collimator.

the validation of thin targets as primary scrapers. A significant increase of the impact parameter in the collimator is observed when the targets become the primary collimator. The signal comming from the detectors located downstream from the collimator is reduced by a factor of two indicating a better absorption in the collimator block (see Fig. 9) and a similar increase of efficiency. The impact parameter measured with the different materials agrees with the calculated value within the errors of the measurement.

\section{ACKNOWLEDGMENTS}

I would like to thank all the SNS accelerator physicists as this is not the work of only one person but the effort of a groups in several laboratories. For the same reason, I owe recognision to Y. Fedotov and all the team in IHEP, A. Drees and D. Gassner. Special thanks to J.B. Jeanneret for his constructive criticism and the CERN staff for invaluable assistance during the experiment at SPS.

\section{REFERENCES}

[1] Y. Papaphilippou, these Proceedings

[2] J.B. Jeanneret Phys. Rev. ST Accel. Beams 1, 081001

[3] P. Bryant in: "Handbook of Accelerator Physics and Engineering", Ed. A.W.Chao, M. Tigner

[4] D. Groom et al. "The review of Particle Physics" The European Physical Journal C15 (2000) 1

[5] N. Catalan-Lasheras, these proceedings

[6] C.M. Warsop. AIP Conf. Proc. 448

[7] R.J. Macek. AIP Conf. Proc. 448

[8] N. Catalan-Lasheras. Phys. Rev. ST Accel. Beams 4, 010101

[9] M. Shirakata et al., EPAC'00 Vienna; C.M. Warsop et al., EPAC'00 Vienna; "The proton Driver Design Study", FERMILAB-TM-2136; S. Ozaki, R.B. Palmer and M.S. Zisman (Ed.)"Feasibility study-II of a Muon-Based Neutrino Source" Draft 2001

[10] J. Wei et al. Phys. Rev. ST Accel. Beams 3, 080101

[11] H. Ludewig et al., PAC 1999, New York, 1999

[12] J.B. Jeanneret and T. Trenkler. CERN SL 94-105 AP.

[13] S. Cousineau, these proceedings

[14] N. Catalan-Lasheras et al., EPAC'98 Stockholm and N. Catalan-Lasheras, Ph.D. Thesis 1999

[15] N. Catalan et al., EPAC'00 Vienna 